\title{
On the Equilibrium States in Quantum Statistical Mechanics
}

\author{
R. HAAG \\ Department of Physics, University of Illinois, Urbana, Illinois* \\ N. M. HugEnHoltz and M. WinNINK \\ Natuurkundig Laboratorium, Rijks-Universiteit, Groningen
}

Received February 15, 1967

\begin{abstract}
Representations of the $C^{*}$-algebra $\mathfrak{A}$ of observables corresponding to thermal equilibrium of a system at given temperature $T$ and chemical potential $\mu$ are studied. Both for finite and for infinite systems it is shown that the representation is reducible and that there exists a conjugation in the representation space, which maps the von Neumann algebra spanned by the representative of $\mathfrak{A}$ onto its commutant. This means that there is an equivalent anti-linear representation of $\mathfrak{A}$ in the commutant. The relation of these properties with the Kubo-Martin-Schwinger boundary condition is discussed.
\end{abstract}

\section{Introduction}

In statistical mechanics one studies large systems and the aim is to derive the macroscopic, or thermodynamical properties of such systems from the equations of motion of the individual particles. Due to their large size, such systems have features such as phase transitions, transport phenomena, which are absent in small systems. To exhibit such features in their purest form one has to consider the limiting case of infinitely large systems, i.e., systems with infinitely many degrees of freedom. The usual formulation of classical or quantum mechanics and of statistical mechanics does not allow the treatment of systems with infinitely many degrees of freedom. This means that one has to consider large, but finite, systems and take the thermodynamical limit at the end. From a logical point of view it seems advantageous to reformulate the theory in such a way that no recourse has to be taken to finite systems. While this aim has not been reached in a complete and satisfactory manner some interesting partial results are known. Among these we may count the Kubo-Martin-Schwinger boundary condition for "thermodynamic Green's functions" [1] and [2], the properties found by ArAKI and

* Present address: II. Institut für theoretische Physik, 2 Hamburg 50, Luruper Chaussee 149, Germany. 
Woops [3] for the equilibrium state of a free Bose gas and the decomposition theory of invariant states of infinite systems [4], [5], [6].

The main objective of the present paper is to show that certain properties of the equilibrium state which were found by ARAKI and Woops for the free Bose gas have general validity for quantum statistical systems and to exhibit the relationship of these properties to the Kubo-Martin-Schwinger boundary condition.

The following attitude will be taken: a state of an infinite system is characterized by the expectation values of quasilocal (macroscopically local) observables in the state. In other words, a state is described by an expectation functional over an algebra $\mathfrak{A}$ whose elements correspond to quasilocal physical operations on the system. This description of states has been widely used in the treatment of infinitely extended quantum systems (field theory, or many body problem) ${ }^{1}$.

Among all the possible states of a system, we must single out the equilibrium states. Here we shall dodge all fundamental questions and adopt the pragmatic definition of an equilibrium state as the limit of a sequence of Gibbs grand canonical ensembles of increasing volume.

In section II we describe the relevant mathematical structure and discuss some conditions for the existence of the infinite volume limit and its insensitivity to surface effects. An easy consequence is then the property (3.32) which is equivalent to the Kubo-Martin-Schwinger boundary condition.

Each state (expectation functional) determines in a natural manner a representation of the algebra $\mathfrak{A}$ by operators in a Hilbert-space ${ }^{2}$. We use the following notation. The elements of the algebra are denoted by $A, B$ etc. A representation of the algebra is written as $R(\mathfrak{U})$. Specifically, $R(A)$ is the operator representing the element $A$. If $\omega$ is a state then $\omega(A)$ denotes the expectation value of $A$ in this state. Starting from the state the GNS-construction gives us a representation $R_{\omega}(\mathfrak{Z})$ in a Hilbert space $\mathfrak{H}_{\omega}$ and a vector $\Omega$ in $\mathfrak{G}_{\omega}$ with the property

$$
\omega(A)=(\Omega, R(A) \Omega)
$$

for all $A \in \mathfrak{A}$. It also gives us a whole family of other states

$$
\omega_{\psi}(A)=(\Psi, R(A) \Psi)
$$

where $\Psi$ is any vector in $\mathfrak{G}_{w}$. The states $\omega_{\psi}$ which belong to the same family may be pictured physically as states which differ essentially only in finite regions from each other.

1 See e.g. [7] where many references may be found.

2 This standard construction will be called the GNS-construction after I. M. Gel'Fand, M. A. Natmark and I. E. Segal who share the credit for the development of many of the mathematical concepts used. A simple survey of the use of these ideas in physics may be found in [7]. 
We can now state those properties of an equilibrium state, demonstrated in [3] and [8] for the free Bose- und Fermi gas, with which we will be concerned:

i) The state is impure, i.e., the representation $R$ is reducible.

ii) There is a symmetry between the von Neumann algebra generated by $R(\mathfrak{A})$ and its commutant. Specifically, there is an antiunitary conjugation operator $J$ such that

and ${ }^{3}$

$$
J R(A) J^{-1}=S(A) ; \quad J^{-1}=J
$$

$$
\{S(\mathfrak{U})\}^{\prime}=\{R(\mathfrak{U})\}^{\prime \prime} ;
$$

iii) If $h(x)$ denotes the algebraic expression for the energy density then the generator of the time translations in $\mathfrak{S}_{\omega}$ is formally given by

and not by

$$
\int(R(h(x))-S(h(x))) d^{3} x
$$

$$
\int R(h(x)) d^{3} x
$$

The precise meaning of the formal expression (1.5) will become clear in the following sections. The formal expression (1.6) can never be defined in a meaningful way for the cases of interest in infinite systems.

The properties (i), (ii) and (iii) above already hold for a Gibbs ensemble in a finite volume. This will be discussed in Section III. Section IV will deal with the infinite system and show the relation between these properties and the Kubo-Martin-Schwinger condition.

\section{Assumptions and Notation}

For simplicity we consider a system with one component (only one type of particle). Let $\Psi^{*}(x)$ be the creation operator of a particle at the point $x$ at the time $t=0$ and $\Psi(x)$ the corresponding destruction operator. These objects satisfy the canonical commutation (or anti-commutation) relations

$$
\left[\Psi(x), \Psi^{*}(y)\right]_{ \pm}=\delta^{3}(x-y) ; \quad[\Psi(x), \Psi(y)]_{ \pm}=0 .
$$

We could regard $\Psi$ and $\Psi^{*}$ as abstract algebraic quantities but it is more convenient to interpret them as operators in the standard Fock space $\mathfrak{S}_{F}$. More precisely, this means that for every square integrable function $f$ we have operators $\Psi(f), \Psi^{*}(f)$ in a Hilbert space $\mathfrak{G}_{F}$. These operators are adjoints of each other and related to $\Psi(x), \Psi^{*}(x)$ symbolically by

$$
\Psi^{*}(f)=\int \Psi^{*}(x) f(x) d^{3} x ; \quad \Psi(f)=\int \Psi(x) f^{*}(x) d^{3} x,
$$

${ }^{3}$ If $R$ is an algebra of bounded operators in a Hilbert space then $R^{\prime}$ denotes its commutant, i.e. the set of all bounded operators which commute with every operator in $R$. The "bicommutant" $R$ " is equal to the weak closure of $R$. 
i.e. $\Psi *(f)$ depends linearly on $f$. The commutation relations (2.1) read then

$$
[\Psi(g), \Psi *(f)]_{ \pm}=(g, f)
$$

In $\mathfrak{S}_{F}$ there is one vector $\Omega_{0}$ (physically the zero particle state) determined by

$$
\Psi(f) \Omega_{0}=0 \text { for all } f .
$$

The Hilbert space is then generated by repeated application of the $\Psi^{*}(f)$ on $\Omega_{0}$.

Let $V$ be any finite volume. We define $\mathfrak{A}(V)$ as the von Neumann algebra determined by all the $\Psi(f), \Psi^{*}(f)$ with functions $f$ having support in $V$.

Clearly $\mathfrak{A}\left(V_{1}\right)$ commutes with $\mathfrak{A}\left(V_{2}\right)$ if $V_{1} \cap V_{2}$ is empty. Also, due to (2.3) and (2.4) we may define the subspace

$$
\mathfrak{H}_{F^{\prime}}^{(V)}=\mathfrak{A}(V) \Omega_{0}
$$

$\mathfrak{S}_{F}^{(V)}$ is an irreducible representation space of $\mathfrak{A}(V)$ and the space $\mathfrak{S}_{F}$ may be factored into

$$
\mathfrak{S}_{F}=\mathfrak{S}_{F}^{(V)} \times \mathfrak{S}_{F}^{\prime}
$$

$\mathfrak{A}(V)$ is a weakly closed set of bounded operators. It is a forteriori closed in the topology of the operator norms $\|A\|$, i.e. $\mathfrak{A}(V)$ is a $C^{*}$-algebra. We define now the $C^{*}$-algebra $\mathfrak{A}$ as the closure in the norm topology of the union of all $\mathfrak{A}(V)$ remembering that $V$ always denotes a bounded region:

$$
\mathfrak{U}=\overline{\cup \mathfrak{A}(V)} \text {. }
$$

Some explanation is in order concerning the motivation of these definitions. We have specified a family of $C^{*}$-algebras and shall ultimately be concerned with the representation of $\mathfrak{A}$ in a Hilbert space $\mathfrak{H}_{\omega}$ (see introduction). This representation must be clearly inequivalent to the one in $\mathfrak{S}_{F}$ because all state vectors in $\mathfrak{F}_{F}$ give a decreasing probability for very large particle number whereas all states in $\mathfrak{G}_{\omega}$ describe an actually infinite particle number (finite mean density). However, the specification of $\mathfrak{A}(V)$ as a von Neumann algebra in $\mathfrak{S}_{F}$ means that as far as the observables in a finite volume are concerned the representations in $\mathfrak{G}_{F}$ and in $\mathfrak{H}_{\omega}$ must be quasiequivalent in the sense of Mackey. In physical terms this means that the particle number in a finite volume is a meaningful quantity and that we shall never consider representations of the commutation relation $(2.1)$ in which

is not definable ${ }^{4}$.

$$
N_{V}=\int_{V} \Psi^{*}(x) \Psi(x) d^{3} x
$$

\footnotetext{
4 Compare [9] for a discussion of this point.
} 
For the observables in an infinite volume on the other hand it is essential to exclude the truly global quantities (such as total energy, total particle number). Therefore we cannot take the algebra of all bounded operators in $\mathfrak{G}_{F}$ (which could result as the weak closure of $\cup \mathfrak{A}(V)$ ) as our total algebra $\mathfrak{A}$ but must restrict ourselves to the quasilocal quantities, which can be approximated in the norm topology by operators belonging to finite regions.

The dynamics of the system is characterized by a Hamiltonian $H$. This Hamiltonian is a well defined operator in $\mathfrak{G}_{F}$ and the same is true for the total particle number $N$. Since we will be concerned with Gibbs ensembles, the combination

$$
H^{\prime}=H-\mu N
$$

(where $\mu$ is a given number, the chemical potential) will be of special importance and we shall call the unitary operator

$$
U(t)=e^{i H^{\prime} t}=e^{i(H-\mu N) t}
$$

for brevity the time translation operator. The following assumptions have to be made about $H^{\prime}$ :

a) For every volume $V$ one can introduce an ${H^{\prime}}_{V}$ so that

$$
U_{V}(t)=e^{i H^{\prime} v(t)}
$$

belongs to $\mathfrak{A}(V)$. This family of "partial Hamiltonians" shall be approximately additive i.e. if a volume $V$ is partioned into two parts $V_{1}$ and $V_{2}$ then

$$
H^{\prime}{ }_{V}-H_{V_{1}}^{\prime}-H_{V_{2}}^{\prime}=H_{s}^{\prime}
$$

shall be essentially a surface term.

More precisely we shall assume that for fixed $A$ and fixed $t$ the transforms

$$
A_{t}^{V_{n}}=U_{V_{n}}(t) A U_{V_{n}}^{-1}(t)
$$

form a Cauchy sequence in the norm topology if

$$
V_{n+1} \supset V_{n} \text { and } \cup V_{n}=R^{3} \text { (the whole space). }
$$

The limit $n \rightarrow \infty$ therefore exists and belongs to $\mathfrak{A}$.

The same statement will continue to hold, if $A$ is any element of $\mathfrak{A}$ because $A$ can then still be arbitrarily closely approximated by an element from some $\mathfrak{A}(V)$. It is now clear in which sense $U_{V}(t)$ should approximate $U(t)$ : For every $A \in \mathfrak{A}$ and every sequence of volumes $V_{n}$ satisfying (2.14)

$$
A_{t}=U(t) A U^{-1}(t)=\lim _{n \rightarrow \infty} U_{V_{n}}(t) A U_{V_{n}}^{-1}(t) .
$$

In other words, $U(t)$ defines an automorphism $A \rightarrow A_{t}$ of the algebra of quasilocal operators and this automorphism is the limit in the norm topology of those induced by $U_{V_{n}}(t)$. 
Note that there is no uniform estimate

$$
\left\|A_{t}-A_{t}^{\nabla_{n}}\right\|<\varepsilon\|A\|
$$

for all $A \in \mathfrak{A}\left(V_{0}\right)$. A counter example against this assumption is obtained if we choose $A$ to be the observable measuring the number of particles in $V_{0}$ with velocities between $v_{1}$ and $v_{2}$ and consider for simplicity a state with only one particle in the universe. If $v_{1} t$ is greater than the linear dimensions of $V_{0}$ then the motion given by $H^{\prime}{ }_{V}$ and that given by $H^{\prime}$ differ drastically since in the first case we have a reflection at the boundary of $V_{0}$ and not in the second case. A consequence of this comment is that for two adjoining regions $V_{1}, V_{2}$ such that $V_{1} \cup V_{2}=V_{3}$ the operator $U^{V_{3}}(t) U^{V_{2}}(t)^{-1} U^{V_{1}}(t)^{-1}$ cannot be approximated in the norm topology by an operator from a surface layer around the interface of $V_{1}, V_{2}$.

b) The interparticle forces must saturate. This requires that for every $V$, every positive $\beta$ and a certain range of $\mu$-values the operator $e^{-\beta H^{\prime} v}$, considered as an operator in $\mathfrak{G}_{F}^{(V)}$ should have a finite trace ${ }^{5}$. This being the case, one can define the Gibbs state for given $\beta, \mu, V$ by

where

$$
\omega_{V}(A)=\operatorname{Tr}_{V}\left(\varrho_{V} A\right)
$$

$$
\varrho_{V}=\left(\operatorname{Tr}_{V} e^{-\beta H^{\prime} V}\right)^{-1} e^{-\beta H^{\prime} V}
$$

and where $\operatorname{Tr}_{V}$ denotes the trace in the Hilbert space $\mathfrak{G}_{F}^{(V)}$.

This is a well defined expectation functional for all $A \in \mathfrak{A}(V)$ because, if $A$ is a bounded operator and $\varrho$ has a finite trace then also $\varrho A$ has a finite trace.

c) The next problem concerns the limit $V \rightarrow \infty$. Let $V_{n}$ again be a set of regions of increasing size as used in (2.14) and let $A \in \mathfrak{A}\left(V_{0}\right)$. It is expected that under normal circumstances the numerical sequence $\omega_{V_{n}}(A)$ converges:

$$
\lim _{n \rightarrow \infty} \omega_{V_{n}}(A)=\omega(A) .
$$

This will be assumed to hold for any set of regions satisfying (2.14) as long as $A$ belongs to the algebra of a bounded region. Then (2.18) uniquely defines a state over the whole algebra because for $A \in \cup \mathfrak{A}(V)$

$$
\omega\left(A^{*} A\right)>0 ; \quad|\omega(A)| \leqq\|A\| .
$$

These properties are evident because they hold for every $\omega_{V_{n}}$.

They allow the extension of the definition of $\omega(A)$ from the operators of bounded regions to those of $\mathfrak{A}$.

5 The conditions on the potential which are imposed by this requirement have been extensively studied by D. Ruelle [10]. Recently F. J. Dyson [11] proved that the Coulomb forces between equal numbers of positively and negatively charged particles saturate. 
The later sections of this paper will be based on assumptions a), b) and c). Of these the first two appear quite satisfactory as basic assumptions since they are related rather directly to important properties of the interparticle forces, namely the sufficiently fast decrease of these forces for large distances and the saturation. The property c) is on rather different footing. We know that it must fail for special values of $\beta, \mu$ (phase transition points). Hence a study of its relation to simpler properties of the system is of great interest. No such discussion will be attempted here, however.

\section{Some Properties of the Gibbs States}

In this section we shall be concerned only with a fixed, finite volume $V$ and the Gibbs state defined by (2.16), (2.17). We shall omit therefore, in this section indices $V$ and write $\mathfrak{A}$ instead of $\mathfrak{A}(V), \omega$ instead of $\omega_{V}$ etc.

As described in the introduction we can use the expectation functional $\omega$ to construct a Hilbert space $\mathfrak{S}_{\omega}$ in which we have a cyclic representation of $\mathfrak{A}$ by operators $R(A)$ and a cyclic vector $\Omega$ corresponding to the state $\omega$. We shall now give an explicit description of this representation. Since the density matrix $\varrho$ is a positive operator with finite trace, the operator

$$
x_{0}=\varrho^{\frac{1}{2}} \text {. }
$$

is well defined. It has the properties ${ }^{6}$

1. $x_{0}$ is a Hilbert-Schmidt operator.

2. For all $A \in \mathfrak{A}$

$$
\begin{array}{lll}
A \varkappa_{0}=0 & \text { implies } & A=0 \\
\varkappa_{0} A=0 & \text { implies } & A=0 .
\end{array}
$$

Proof of property 2. $\varrho^{-1}=\left(\operatorname{Tr} e^{-\beta H^{\prime}}\right) e^{\beta H^{\prime}}$ is an unbounded positive operator with a dense domain in $\mathfrak{G}_{F}$. The same holds for $\varkappa_{0}^{-1}$. Consequently $x_{0}$ has a dense range in $\mathfrak{G}_{F}$. This is the same statement as $(3.2 \mathrm{a}) .(3.2 \mathrm{~b})$ follows then by Hermitian conjugation.

Consequence:

$$
A \in \mathfrak{A} \text { and } \omega\left(A^{*} A\right)=0 \text { imply } A=0 .
$$

Here $\omega$ is defined by (2.16) which can be written as

$$
\omega(A)=\operatorname{Tr}\left(\varkappa_{0} A \varkappa_{0}\right) .
$$

Let $\mathfrak{R} \subset \mathfrak{A}$ be the algebra of Hilbert-Schmidt operators. This algebra has a number of well known properties:

a) $\mathfrak{R}$ is a 2 -sided ideal of $\mathfrak{A}$, i.e. $\mathfrak{A} \Re=\Re ; \mathfrak{R}=\mathfrak{R}$.

6 The Hilbert-Schmidt class consists of those operators whose absolute square have a finite trace.

16 Commun. math. Phys., Vol. 5 
b) $\Omega$ may be considered as a linear space equipped with a scalar product

$$
\left(x, x^{\prime}\right)=\operatorname{Tr}\left(x^{*} x^{\prime}\right) .
$$

One may introduce a norm $\|x\| \|$ derived from this scalar product by

$$
\|x\|^{2}=(x, x)
$$

and finds that $\Omega$ is closed in the topology defined by this norm. Thus one can regard $\mathfrak{R}$, equipped with this norm as either a Banach algebra or as a Hilbert space. The relations

$$
\left(x_{1} x_{2}, x_{3}\right)=\left(x_{2}, x_{1}^{*} x_{3}\right) ; \quad\left(x, x^{\prime}\right)=\left(x^{\prime *}, x^{*}\right)
$$

make $\Re$ into a Hilbert algebra .

We shall now use the symbol $\mathfrak{H}$ instead of $\Re$ to denote the Hilbert space whose vectors are the Hilbert-Schmidt operators of $\mathfrak{G}_{F}$, the scalar product being defined by (3.5). Consider the following two representations of $\mathfrak{A}$ by operators in $\mathfrak{S}$ :

1. We represent $A \in \mathfrak{A}$ by the operator $R(A)$ in $\mathfrak{G}$ defined by

$$
R(A) x=A x .
$$

2. We represent $A \in \mathfrak{A}$ by the operator $S(A)$ in $\mathfrak{S}$ defined by

$$
S(A) x=x A^{*} \text {. }
$$

If $\|R(A)\|$ and $\|S(A)\|$ denote the operator norms in $\mathfrak{G}$ one easily checks that

because $^{8}$

$$
\|R(A)\|=\|S(A)\|=\|A\|
$$

Also

$$
\|R(A)\|^{2}=\sup \frac{\left.\|A \varkappa\|\right|^{2}}{\|x\|^{2}}=\sup \frac{\operatorname{Tr} \varkappa^{*} A^{*} A \varkappa}{\operatorname{Tr}\left(\varkappa^{*} \varkappa\right)}=\|A\|^{2} .
$$

$$
\begin{aligned}
R\left(A_{1}\right) R\left(A_{2}\right) & =R\left(A_{1} A_{2}\right) ; \\
R\left(\lambda_{1} A_{1}+\lambda_{2} A_{2}\right) & =\lambda_{1} R\left(A_{1}\right)+\lambda_{2} R\left(A_{2}\right) ; R\left(A^{*}\right)=(R(A))^{*} \\
S\left(A_{1}\right) S\left(A_{2}\right) & =S\left(A_{1} A_{2}\right) ; S\left(\lambda_{1} A_{1}+\lambda_{2} A_{2}\right)=\bar{\lambda}_{1} S\left(A_{1}\right)+\bar{\lambda}_{2} S\left(A_{2}\right) ; \\
S\left(A^{*}\right) & =(S(A))^{*} .
\end{aligned}
$$

Hence $R$ is an ordinary *-representation, $S$ is a conjugate (antilinear)*representation of $\mathfrak{A}$. We have

$$
\{R(\mathfrak{Q})\}^{\prime \prime}=\{S(\mathfrak{A})\}^{\prime} .
$$

Here the prime indicates the commutant, the double prime the commutant of the commutant (which, by a famous theorem of von Neumann coincides with the weak closure).

7 See e.g. [12].

8 The last identity in (3.11) follows because we can choose $x$ as the projection operator on a state in $\mathfrak{G}_{F}$ which is an almost eigenvector of $A * A$ to the highest spectral value. 
Proof. Since

We have

$$
R(A) S(B) \varkappa=A \varkappa B^{*}=S(B) R(A) \varkappa .
$$

$$
R(\mathfrak{A}) \subset\{S(\mathfrak{A})\}^{\prime},
$$

or, taking the weak closures on both sides

$$
\{R(\mathfrak{U})\}^{\prime \prime} \subset\{S(\mathfrak{U})\}^{\prime} .
$$

On the other hand, since the subalgebra $\Omega \subset \mathfrak{A}$ is a Hilbert algebra one has the well-known theorem [12]

$$
\{R(\mathfrak{R})\}^{\prime \prime}=\{S(\Omega)\}^{\prime} .
$$

Obviously $R(\mathfrak{U}) \supset R(\mathfrak{R}) ; \quad S(\mathfrak{U}) \supset S(\mathfrak{R})$ and hence $\{S(\mathfrak{A})\}^{\prime} \subset\{S(\mathfrak{R})\}^{\prime}$. Therefore (3.13), (3.14) together imply

$$
\{R(\mathfrak{R})\}^{\prime \prime}=\{R(\mathfrak{A})\}^{\prime \prime}=\{S(\mathfrak{R})\}^{\prime}=\{S(\mathfrak{U})\}^{\prime}
$$

which proves the theorem.

Theorem 2. The vector $\varkappa_{0}$ is cyclic for both representations and

$$
\omega(A)=\left(\varkappa_{0}, R(A) \varkappa_{0}\right)=\overline{\left(\varkappa_{0}, S(A) \varkappa_{0}\right)} .
$$

The representations $R$ and $S$ are transformed into each other by an antiunitary conjugation operator $J$ :

$$
J R(A) J^{-1}=S(A) ; \quad J=J^{-1} ; \quad J \varkappa_{0}=\varkappa_{0} .
$$

Proof. The cyclicity of $\varkappa_{0}$ for the representation $R$ is related to (3.3) in the following way: Suppose there is a $x \in \mathfrak{S}$ such that $\left(\varkappa, R(A) \varkappa_{0}\right)=0$ for all $A \in \mathfrak{A}$. Since $\varkappa$ can also be regarded as an element of $\mathfrak{A}$ we can choose $A=\varkappa x_{0}$. Then we would get $\operatorname{Tr} x^{*} \varkappa_{0}^{2}=\omega\left(x^{*} \varkappa\right)=0$ and hence by $(3.3) x=0$.

The first part of eq. (3.16) results directly from the defining equations (3.4), (3.5), (3.8). This means that the representation $R$ is the one resulting from the GNS-construction with the state $\omega$.

The operator $J$ is defined by

$$
J \varkappa=x^{*}
$$

$J$ is anti-unitary, since

$$
\left(J x_{1}, J x_{2}\right)=\left(x_{1}^{*}, x_{2}^{*}\right)=\operatorname{Tr} x_{1} \varkappa_{2}^{*}=\left(x_{2}, x_{1}\right) .
$$

Because $J^{2}=1$ the operator $J$ is a conjugation. The remaining statements in theorem 2 are now immediate consequences.

Next we consider the automorphism group on the algebra which is induced by the time translations:

$$
A \rightarrow A_{t}=U(t) A U^{-1}(t) .
$$

We want to implement these automorphism in the representation $R$ by unitary operators $\hat{U}(t) \in \mathfrak{I}(\mathfrak{S})$. In other words, the unitary operators $\hat{U}$ should satisfy

$$
\hat{U}(t) R(A) \hat{U}^{-1}(t)=R\left(A_{t}\right)
$$


It is clear that (3.20) alone does not define the $\hat{U}(t)$ uniquely. The general solution of $(3.20)$ is

$$
\hat{U}(t)=R(U(t)) \cdot V(t)
$$

where $V$ is an arbitrary unitary operator from $\{R(\mathfrak{U})\}^{\prime}$. The expectation functional $\omega$ is invariant under time translation i.e.

$$
\omega\left(A_{t}\right)=\omega(A) \text {. }
$$

Therefore it appears natural to choose $\hat{U}(t)$ in such a way that the vector $x_{0} \in \mathcal{S}$ which represents the state $\omega$ is invariant under $\hat{U}(t)$ :

$$
\hat{U}(t) \varkappa_{0}=\varkappa_{0} \text {. }
$$

This fixes the choice of $V$ in (3.22) uniquely and we get (due to the fact that $U(t)$ and $\varkappa_{0}$ commute)

$$
\hat{U}(t)=R(U(t)) \cdot S(U(t))
$$

which corresponds to the (somewhat symbolic) expression for the generator:

$$
\hat{H}^{\prime}=R\left(H^{\prime}\right)-S\left(H^{\prime}\right)
$$

In the case of the finite system the requirement (3.23), while very natural, may still appear to be somewhat arbitrary. For the infinite system, where $U(t)$ is no longer an element of $\mathfrak{A}$ the fact that the automorphism is unitarily implementable at all depends crucially on the invariance of the state $\omega$ (eq. (3.22)). From this one derives that a unitary operator $\hat{U}(t)$ with the properties (3.20), (3.23) can be constructed and is uniquely defined by these properties in the space $\mathfrak{H}_{\omega}$. On the other hand the quantity $R(U(t))$ is undefined and undefinable in that case.

One other way to understand (3.25) is to note that the vector $x_{0}$ representing the Gibbs state is not an eigenstate of the energy operator $R\left(H^{\prime}\right)$ because the energy in a Gibbs ensemble is not sharply defined. In an infinite system the fluctuation of the energy is infinite and therefore $R\left(H^{\prime}\right)$ cannot be defined at all any more. The equilibrium state is, however, an eigenstate of $\hat{H}^{\prime}$.

We add two remarks. The first remark concerns the connection between our results and those obtained by ARAKI and Woods for the ideal Bose gas. They find a representation of $\mathfrak{A}$ in the commutant and not, as we find, a conjugate representation. They also find a unitary operator $\hat{U}(t)$, which implements the automorphisms of time translations. However, in their case, the representatives of $\mathfrak{A}$ in the commutant run backward under the transformation $\hat{U}(t)$. It is clear that there is a simple connection between the two descriptions: a time reversal of the commutant which transforms a representation into a conjugate representation. 
The second point concerns the cyclic vector. Our proof that $\varkappa_{0}$ is cyclic for $R(\mathfrak{U})$ and for $S(\mathfrak{U})$ is based on the property of $\varkappa_{0}$ that $A \varkappa_{0}=0$ implies $A=0$ for all $A \in \mathfrak{A}$.

This property holds for a large class of Hilbert-Schmidt operators, in particular for those corresponding to all possible equilibrium states. This means that as long as $V$ is finite our representation contains among others the equilibrium states for all $\beta$ and $\mu$.

To close this section we shall write down the KMS-boundary condition in a form which is suitable for our objective in section IV, namely the discussion of the thermodynamic limit $V \rightarrow \infty$. If $z=t+i \gamma$ is a complex number and $A \in \mathfrak{A}$ then

$$
A_{z}=e^{i H^{\prime} z} A e^{-i H^{\prime} z}
$$

will, in general, not be a bounded operator. But

$$
A_{z} e^{-i \beta H^{\prime}}=e^{i H^{\prime} t} e^{-H^{\prime} \gamma} A e^{-i H^{\prime} t} e^{-H^{\prime}(\beta-\gamma)}
$$

will be bounded and of trace class for $0 \leqq \gamma \leqq \beta$ because, with these restrictions on $\gamma$, all the factors in the above product are bounded and either the second factor or the last (or both) are of trace class.

Similarly, $e^{-\beta H^{\prime}} A_{z}$ will be of trace class for $-\beta \leqq \gamma \leqq 0$. Therefore, for any $A, B \in \mathfrak{A}$ the function

$$
F_{A B}(z)=\operatorname{Tr} B A_{z} e^{-\beta H^{\prime}}
$$

is well defined in the strip $0 \leqq \gamma \leqq \beta$. In fact, it is differentiable, hence analytic, in the open strip $0<\gamma<\beta$ and continuous at the boundaries. This statement follows from the fact that $H^{\prime} e^{-\alpha H^{\prime}}$ is a bounded operator for any $\alpha>0$.

Similarly, the function

$$
G_{A B}(z)=\operatorname{Tr} e^{-\beta H^{\prime}} A_{z} B
$$

is analytic in the strip $-\beta<\gamma<0$ and continuous at the boundaries. We have, for $\gamma=0$

$$
F_{A B}(t)=\omega\left(B A_{t}\right) ; \quad G_{A B}(t)=\omega\left(A_{t} B\right)
$$

and, from the invariance of the trace under cyclic permutations one gets the KMS-boundary condition

$$
F_{A B}(t+i \beta)=G_{A B}(t) .
$$

For the passage to the thermodynamic limit it is convenient to use the functions $F$ and $G$ only for real times. Therefore, we reexpress the above mentioned properties in the following way: Let $\hat{f}(\varepsilon)$ be a class $\mathscr{D}$ test function in the sense of LAURENT ScHwarTz (infinitely differentiable and with compact support). Define, with $z=t+i \gamma$

$$
f(z)=\int \hat{f}(\varepsilon) e^{i z \varepsilon} d \varepsilon .
$$


Then $f(z)$ is analytic in the entire $z$-plane and $t^{n} f(t+i \gamma)$ is a bounded function of $t$ for any fixed $\gamma$ and positive $n$. Therefore, multiplying (3.30) with $f(t)$ and integrating we can shift the integration path on the left hand side within the analyticity domain of $F_{A B}$ and obtain

$$
\int f(t-i \beta) \omega\left(B A_{t}\right) d t=\int f(t) \omega\left(A_{t} B\right) d t .
$$

The validity of this relation for any $f \in \mathscr{D}$ and any pair $A, B \in \mathfrak{A}$ is equivalent to the KMS-boundary condition. This can be seen in the following way. If $\hat{F}(\varepsilon), \hat{G}(\varepsilon)$ denote the Fourier transforms of $F(t)$ $=\omega\left(B A_{t}\right)$ and $G(t)=\omega\left(A_{t} B\right)$ then (3.31) states that

$$
\hat{F}(\varepsilon)=e^{\beta \varepsilon} \hat{G}(\varepsilon)
$$

where $F$ and $G$ are considered as distributions over $\mathscr{D}$. On the other hand, we know from their definitions that $F(t), G(t)$ are bounded, continuous functions of $t$ and hence $\hat{F}, \hat{G}$ are distributions over $\mathscr{S}$. Since $\mathscr{D}$ is a totalizing subset of $\mathscr{S}$, the relation (3.32) holds for the tempered distributions $\hat{F}, \hat{G}$. This implies the analyticity of the functions $F(z), G(z)$ in the open strips described above. Since $F(t), G(t)$ are bounded, continuous functions, they are, by (3.32) the boundary values of $F(t+i \gamma)$ for $\gamma=0$ resp. for $\gamma=\beta$.

Thus the analyticity statements for $F(z), G(z)$ with KMS-boundary condition (3.30) are equivalent with (3.31).

\section{The Infinite System}

In section III extensive use was made of the fact that the Gibbs states $\omega_{V}$ have the normal form $(2.16),(2.17)$. The states of infinite systems are in general not normal i. e., they cannot be described by a density matrix in $\mathfrak{H}_{F}$. However, under the assumptions of section II one finds that (3.31) and hence the KMS-boundary condition remain valid in the thermodynamic limit. From this relation one can deduce the other general properties of the equilibrium state and the corresponding GNS-representation of $\mathfrak{A}$ which were derived for the Gibbs states of a finite system in section II.

To show the validity of (3.31) in the thermodynamic limit we only have to prove that

$$
\lim _{V \rightarrow \infty} \int \omega_{V}\left(B A_{t}^{V}\right) f(t) d t=\int \omega\left(B A_{t}\right) f(t) d t
$$

for class $\mathscr{S}$ test functions $f(t)$. Here $\omega_{V}$ is the Gibbs state for volume $V$ and $A_{t}^{V}=U_{V}(t) A U_{V}^{-1}(t)$. Since the expectation values in the integrands are uniformly bounded functions of $t$ this is equivalent to

$$
\lim _{V \rightarrow \infty} \omega_{V}\left(B A_{t}^{V}\right)=\omega\left(B A_{t}\right)
$$


for each fixed $t$. To show this we need assumption a) of section II in the form ( $A$ being fixed)

with

$$
\left\|A_{t}^{V}-A_{t}^{V_{1}}\right\|<\varepsilon^{\prime}\left(V_{1}, t\right) \text { for every } \quad V \supset V_{1}
$$

$$
\lim _{V_{1} \rightarrow \infty} \varepsilon^{\prime}\left(V_{1}, t\right)=0,
$$

and we need assumption c) of section II in the form

with

$$
\left|\omega_{V}(C)-\omega(C)\right|<\varepsilon^{\prime \prime}(V, C)
$$

$$
\lim _{V \rightarrow \infty} \varepsilon^{\prime \prime}(V, C)=0 .
$$

Choosing some volume $V_{1} \subset V$ we can write

$$
\begin{aligned}
& \left|\omega_{V}\left(B A_{t}^{V}\right)-\omega\left(B A_{t}\right)\right|=\mid \omega_{V}\left(B A_{t}^{V}\right)-\omega_{V}\left(B A_{t}^{V^{1}}\right)+\omega_{V}\left(B A_{t}^{V^{1}}\right)-\omega\left(B A_{t}^{V^{1}}\right)+ \\
& \quad+\omega\left(B A_{t}^{V_{1}^{1}}\right)-\omega\left(B A_{t}\right) \mid<2 \varepsilon^{\prime}\left(V_{1}, t\right)\|B\|+\varepsilon^{\prime \prime}\left(V, B A_{t}^{V_{1}^{1}}\right)
\end{aligned}
$$

for any choice of $V_{1} \subset V$. Thus we can choose first $V_{1}$ so large that the term involving $\varepsilon^{\prime}$ is smaller than any given positive $\varepsilon$ and then we can choose, keeping $V_{1}$ fixed, a sufficiently large $V$ so that $\varepsilon^{\prime \prime}$ is smaller than $\varepsilon$. This proves (4.1) and therefore the validity of the KMS-boundary condition for the infinite system.

In this section we shall need another condition, which can be shown to be equivalent to the KMS-boundary condition. Let $\widetilde{\mathfrak{U}} \subset \mathfrak{A}$ be the subset of all $A \in \mathfrak{A}$, of which the Fourier-transform $\hat{A}(\varepsilon)$ of $A_{t}$ has compact support ${ }^{9}$. One proves easily that $\widetilde{\mathfrak{U}}$ is a ${ }^{*}$-algebra. $\widetilde{\mathfrak{U}}$ is not empty since all operators of the form $A_{f}=\int d t A_{t} f(t)$ for $\hat{f}(\varepsilon) \in \mathscr{D}$ and $A \in \mathfrak{A}$ belong to $\tilde{\mathfrak{I}}$.

For any $A \in \tilde{\mathfrak{A}}$ we define the mapping $A \rightarrow A_{i x}$ by the formula $A_{i x}=\int d \varepsilon \hat{A}(\varepsilon) \exp (x \varepsilon)$, where $x$ is a real number. A simple calculation shows that this mapping is an automorphism. Since $\left(A_{i x}\right)^{*}=\left(A^{*}\right)_{-i x}$ this automorphism is not a *automorphism. We note that this automorphism commutes with gauge transformations and time translations. The invariance of $\omega(A)$ for time translations implies that $\omega\left(A_{i x}\right)=\omega(A)$ for all $A \in \widetilde{\mathfrak{U}}$.

Let $A$ in (3.31) belong to $\widetilde{\mathfrak{A}}$. In the lefthand side one can again shift the integration path and obtain

$$
\int f(t-i \beta) \omega\left(B A_{t}\right) d t=\int f(t) \omega\left(B A_{t+i \beta}\right) d t .
$$

9 Strictly speaking, the Fourier transform $\hat{A}(\varepsilon)$ is a distribution and not a function of $\varepsilon$. Using the theory of Fourier transforms and convolutions of distributions, one derives the results quoted here in a rigorous way. 
We then get the equation

$$
\int d t f(t)\left[\omega\left(B A_{t+i \beta}\right)-\omega\left(A_{t} B\right)\right]=0
$$

for all $\hat{f}(\varepsilon) \in \mathscr{D}$ and $A \in \hat{\mathfrak{A}}$. Since the expression between brackets is continuous in $t$ the equation holds for any $f \in \mathscr{S}$, so that one gets $\omega\left(B A_{t+i \beta}\right)-\omega\left(A_{t} B\right)=0$ for all $t$. In particular one has

$$
\omega\left(B A_{i \beta}\right)=\omega(A B) \text { for } A \in \widetilde{\mathfrak{U}}, B \in \mathfrak{A} .
$$

If also $B \in \widetilde{\mathfrak{A}}$ one can write

$$
\omega(A B)=\omega\left(B_{-\frac{1}{2} i \beta} A \frac{1}{2} i \beta\right) .
$$

Since the mapping $A \rightarrow A_{\frac{1}{2}} i \beta$ will occur frequently in the following pages we shall use the shorthand notation $A_{b}$ for $A_{\frac{1}{2} i \beta}$. We then have the condition

I.

$$
\omega\left(A_{b} * B_{b}\right)=\omega\left(B A^{*}\right)
$$

for all $A$ and $B \in \widetilde{\mathfrak{A}}$. That condition $\mathrm{I}$ is not only a consequence of but also equivalent to the KMS-boundary condition can be proved without difficulty. Condition (4.3) is basic in the following derivations.

As in the case of the finite system we shall impose the following further condition on $\omega$ :

II.

$$
\omega\left(A^{*} A\right)=0 \text { implies } A=0 \text { for all } A \in \mathfrak{A} .
$$

This condition is stronger than one would like to require on purely physical grounds. Since the subalgebra $\mathfrak{A}_{V}$ for any bounded region $V$ describes a finite system, one would expect the following weaker condition to hold:

$$
\omega\left(A^{*} A\right)=0 \text { implies } A=0 \text { for all } A \in \mathfrak{A}_{V} \text { for some } V \text {. }
$$

However, as we shall see at the end of this section, condition II is a direct consequence of condition $I$, if one assumes that the algebra $\mathfrak{A}$ is simple ${ }^{10}$.

We use standard procedures to construct the representation of $\mathfrak{A}$ defined by $\omega(A)$. As representation space we take the linear space $\mathfrak{A}$. To avoid confusion, the element 1 (the unit), $A_{1}, A_{2}$, etc., when considered as vectors, will be denoted by $\Omega, \Psi_{A_{1}}, \Psi_{A_{2}}$, etc. We introduce the scalar product

$$
\left(\Psi_{A}, \Psi_{B}\right)=\omega(A * B),
$$

${ }_{10}$ A $*$-algebra $\mathfrak{A}$ is simple if it contains no two-sided $*$-ideals different from $\{0\}$. For a proof that the $C^{*}$-algebra of the canonical commutation relations is simple see [13]. 
which defines a norm in the prehilbert space. Its closure is the Hilbertspace $\mathfrak{H}$. Due to condition II, different algebra elements give different vectors.

Another space $\mathfrak{S}^{\prime}$ can be constructed starting from the same linear space $\mathfrak{A}$ with vectors this time denoted by $\Omega^{\prime}, \Psi_{A_{1}}^{\prime}, \Psi_{A_{2}}^{\prime}$, etc., by means of the different scalar product

$$
\left(\Psi_{A}^{\prime}, \Psi_{B}^{\prime}\right)=\omega\left(B A^{*}\right)
$$

Theorem 3. The subspace $\widetilde{\mathfrak{I}}$ is dense in $\mathfrak{G}$ and in $\mathfrak{S}^{\prime}$.

Proof. We shall prove the statement for $\mathfrak{H}$. The proof for $\mathfrak{S}^{\prime}$ is similar. We shall prove that all $A_{f}=\int d t f(t) A_{t}$ with $\hat{f}(\varepsilon) \in \mathscr{D}$ is a dense set in $\mathfrak{Y}$.

Suppose $\left(\chi, \Psi_{A_{f}}\right)=0$ for all $A_{f}$. Now $\left(\chi, \Psi_{A_{f}}\right)=\int d t f(t)\left(\chi, \Psi_{A_{t}}\right)$. Since $\left(\chi, \Psi_{A_{t}}\right)$ is a continuous function of $t$ the integral

$$
\int d t f(t)\left(\chi, \Psi_{A_{t}}\right)=0
$$

for all $f \in \mathscr{S}$ and hence $\left(\chi, \Psi_{A_{t}}\right)=0$ for all $t$. Putting $t=0$ we obtain the equation $\left(\chi, \Psi_{A}\right)=0$ for all $A \in \mathfrak{A}$; but $\mathfrak{A}$ is dense, so that $\chi=0$. This proves that the set $\Psi_{A \mathcal{f}}$ is dense in $\mathfrak{S}$. A fortiori $\widetilde{\mathfrak{A}}$ is dense in $\mathfrak{S}$. For later use we state a somewhat modified version of this theorem.

Theorem 3A. The set $\left\{\Psi_{A}+\Psi_{A_{b}}\right\}$ for all $A \in \widetilde{\mathfrak{U}}$ is dense in $\mathfrak{Y}$.

Proof. We simply repeat the proof of theorem 3 with the only modification that everywhere $f(t)$ is replaced by $f(t)$ is replaced by $f(t)+$ $+f(t+i \beta)$. As one can see easily, the set of all $f(t)+f(t+i \beta)$ with $\hat{f}(\varepsilon) \in \mathscr{D}$ is the same as the set of all $f(t)$ with $\hat{f}(\varepsilon) \in \mathscr{D}$.

We shall use theorem 3 and condition I on $\omega(A)$ to define an isometry between $\mathfrak{S}$ and $\mathfrak{S}$ !

The mapping

$$
\Psi_{A}^{\prime}=S \Psi_{A_{b}} \text { for all } A \in \widetilde{\mathfrak{U}}
$$

maps a dense subspace of $\mathfrak{H}$ onto a dense subspace of $\mathfrak{H}^{\prime} . S$ is an isometric operator since $\left(\Psi_{A}^{\prime}, \Psi_{B}^{\prime}\right)=\omega\left(B A^{*}\right)=\omega\left(A_{b} * B_{b}\right)=\left(\Psi_{A_{b}}, \Psi_{B_{b}}\right)$. Therefore, domain and range of $S$ can be extended to the whole space. We define $\Phi_{A} \in \mathfrak{G}$ for all $A \in \mathfrak{A}$ by

$$
\Phi_{A}=S^{-1} \Psi_{A}^{\prime}
$$

and conclude from (4.6) that

$$
\Phi_{A}=\Psi_{A_{b}}
$$

for all $A \in \widetilde{\mathfrak{A}}$. We also notice that

$$
\left(\Phi_{A}, \Phi_{B}\right)=\omega\left(B A^{*}\right) .
$$


We shall now construct two anti-unitarily equivalent cyclic representations of $\mathfrak{A}$ in the representation space $\mathfrak{H}$.

I. We map $A \in \mathfrak{U}$ onto the operator $R(A)$, defined by

$$
R(A) \Psi_{B}=\Psi_{A B}
$$

This is the well-known GNS-representation.

II. We map $A \in \mathfrak{A}$ onto the operator $S(A)$, defined by

$$
S(A) \Phi_{B}=\Phi_{B A}{ }^{*}
$$

This mapping is a cyclic antilinear *-representation of $\mathfrak{A}$; in particular $\|S(A)\| \leqq\|A\|, S(A) S(B)=S(A B), S(A)^{*}=S\left(A^{*}\right)$ and $S(\lambda A)=\bar{\lambda} S(A)$.

In both representations the positive form $\omega(A)$ is reproduced as an expectation value:

$$
\omega(A)=(\Omega, R(A) \Omega)=\overline{(\Omega, S(A) \Omega)} .
$$

The connection between both representations can again be expressed by means of a conjugation operator. We define the operator $J$ by the equation $J \Psi_{A}=\Phi_{A^{*}}$ for all $A \in \mathfrak{A}$. $J$ has the following simple properties:

1. $J$ is anti-linear.

2. $J$ is norm-conserving and thus anti-unitary.

Proof. $\left\|J \Psi_{A}\right\|^{2}=\left(\Phi_{A^{*}}, \Phi_{A^{*}}\right)=\omega\left(A^{*} A\right)=\left\|\Psi_{A}\right\|^{2}$.

We can, therefore, extend the domain of $J$ to $\mathfrak{g}$.

3. $J^{2}=1$, and hence $J$ is a conjugation.

Proof. Let $A \in \widetilde{\mathfrak{U}} ; J^{2} \Psi_{A}=J \Phi_{A^{*}}=J \Psi_{\left(A^{*}\right)_{b}}=J \Psi_{\left(A_{-b}\right)^{*}}=\Phi_{A_{-b}}=\Psi_{A}$; hence $J^{2}=1$ on a dense set.

4. $J R(A) J=S(A)$.

Proof. $J R(A) J \Phi_{B}=J R(A) \Psi_{B^{*}}=J \Psi_{A B^{*}}=\Phi_{B A^{*}}=S(A) \Phi_{B}$.

It is important to notice at this point that for the existence of these two representations condition I of $\omega(A)$ is not necessary. We only used the fact that there is an isometry between the two Hilbert spaces $\mathfrak{H}$ and $\mathfrak{S}^{\prime}$, and such an isometry always exists. The existence of the special isometry (4.6) which leads to (4.8) is a result of condition I. It is in particular the relation (4.8) valid for a dense set in $\mathfrak{G}$ which is responsible for the following theorem:

Theorem 4.

$$
\{R(\mathfrak{U})\}^{\prime \prime}=\{S(\mathfrak{U})\}^{\prime}
$$


This theorem is the analogue of theorem 1 of the last section. There the proof was based on properties of Hilbert algebras. Although we do not have a Hilbert algebra in the present case ${ }^{11}$, the proof of theorem 4 is very analogous to that for Hilbert algebras ${ }^{12}$. We divide the proof in a number of lemmas.

With each algebra element $A$ there correspond two bounded operators $R(A)$ and $S(A)$. Since $A$ is also a vector in $\mathfrak{S}$, it is natural to extend this correspondence to the whole Hilbert space, and to associate with every vector $\chi \in \mathfrak{S}$ operators $R(\chi)$ and $S(\chi)$ which are defined as follows:

$$
R(\chi) \Phi_{B}=S\left(B^{*}\right) \chi \quad \text { and } \quad S(\chi) \Psi_{B}=R(B) \chi .
$$

These operators are in general not bounded. As special cases we mention the operators $R\left(\Phi_{A}\right)$ and $S\left(\Psi_{A}\right)$ with the properties

$$
R\left(\Phi_{A}\right) \Phi_{B}=\Phi_{A B} \quad \text { and } \quad S\left(\Psi_{A}\right) \Psi_{B}=\Psi_{B A}
$$

These operators are again in general unbounded. That the set of operators $R(\chi)$ and $S(\chi)$ contain $R(A)$ and $S(A)$ as special cases follows from the following lemmas.

Lemma 1. $R\left(\Psi_{A}\right)=R_{A}$ and $S\left(\Phi_{A}\right)=S\left(A^{*}\right)$ for all $A \in \widetilde{\mathfrak{A}}$.

Proof. We prove the first statement; the second one goes similarly. Let $A$ and $B \in \widetilde{\mathfrak{A}}$. Then $R\left(\Psi_{A}\right) \Psi_{B}=R\left(\Psi_{A}\right) \Phi_{B_{-b}}=S\left(B_{-b}{ }^{*}\right) \Psi_{A}$ $=S\left(B_{-b}{ }^{*}\right) \Phi_{A_{-b}}=\Phi_{A_{-b} B_{-b}}=\Psi_{A B}=R(A) \Psi_{B}$. Hence $R\left(\Psi_{A}\right)=R(A)$.

Lemma 2. $R\left(\Psi_{A}\right)=R(A)$ and $S\left(\Phi_{A}\right)=S\left(A^{*}\right)$ for all $A \in \mathscr{A}$.

Proof. Let $B \in \widetilde{\mathfrak{A}}$; then $R\left(\Psi_{A}\right) \Psi_{B}=R\left(\Psi_{A}\right) \Phi_{B_{-b}}=S\left(B_{-b}{ }^{*}\right) \Psi_{A}$ $=S\left(\Phi_{B_{-b}}\right) \Psi_{A}=S\left(\Psi_{B}\right) \Psi_{A}=\Psi_{A B}=R(A) \Psi_{B}$. Hence $R\left(\Psi_{A}\right)=R(A)$.

Lemma 3. $R(\mathfrak{U}) \subset\{S(\mathfrak{U})\}^{\prime}$ and $S(\mathfrak{U}) \subset\{R(\mathfrak{U})\}^{\prime}$.

Proof. From (4.11) we deduce that $S(A) R(\chi) \Phi_{B}=S(A) S\left(B^{*}\right) \chi$ and $R(\chi) S(A) \Phi_{B}=R(\chi) \Phi_{B A} *=S\left(A B^{*}\right) \chi=S(A) S\left(B^{*}\right) \chi$. Hence $[S(A), R(\chi)] \Phi_{B}=0$ and thus $[S(A), R(\chi)]=0$. Using lemma 2 we conclude that $[S(A), R(B)]=0$ for all $A$ and $B \in \mathfrak{A}$, which proves the lemma.

Definition. $M$ is the set of all bounded $R(\chi)$ and $N$ is the set of all bounded $S(\chi)$.

Iemma 4. $M=\{S(\mathfrak{U})\}^{\prime}$ and $N=\{R(\mathfrak{A})\}^{\prime}$.

11 Another and more concise proof of theorem 4 can be given by showing that the subalgebra $\tilde{\mathfrak{A}}$ is a quasi Hilbert algebra [12]. This has been pointed out to us by D. KastLer.

12 See e.g. [12]. 
Proof. We know that $M \subset\{S(\mathfrak{A})\}^{\prime}$. Let $O \in\{S(\mathfrak{A})\}^{\prime} . O \Phi_{B}=O S\left(B^{*}\right) \Omega$ $=S\left(B^{*}\right) O \Omega=R(O \Omega) \Phi_{B}$, where the last equality follows from (4.11). Hence $O=R(O \Omega)$ and thus $O \in M$. We conclude that $\{S(\mathfrak{R})\}^{\prime} \subset M$, which proves the lemma.

Lemma 5. $\left[R(\chi), S\left(\chi^{\prime}\right)\right]=O$ for all bounded $R(\chi)$ and $S\left(\chi^{\prime}\right)$.

Proof ${ }^{13}$. We prove this lemma in the following steps:

i. Let $\chi^{*}=R(\chi)^{*} \Omega$; then $\left(\chi, \Phi_{A}\right)=\left(R(\chi) \Omega, S\left(A^{*}\right) \Omega\right)=(S(A) \Omega$, $\left.R(\chi)^{*} \Omega\right)=\left(\Phi_{A^{*}}, \chi^{*}\right)=\left(J \chi^{*}, \Psi_{A}\right)$.

ii. Since the set $\Psi_{A}+\Phi_{A}$ for all $A \in \widetilde{\mathfrak{A}}$ is dense in $\mathfrak{G}$, there exists a sequence $\left\{A_{n}\right\}$ such that $\chi+J \chi^{*}=\lim _{n \rightarrow \infty}\left(\Psi_{A_{n}}+\Phi_{A_{n}}\right)$; we shall prove that $\chi=\lim _{n \rightarrow \infty} \Psi_{A_{n}}$ and $J \chi^{*}=\lim _{n \rightarrow \infty} \Phi_{A_{n}}$. Let $A \in \tilde{\mathfrak{A}} ;\left\|\Psi_{A}+\Phi_{A}\right\|^{2}$ $=\left\|\Psi_{A}\right\|^{2}+\left\|\Phi_{A}\right\|^{2}+\left(\Psi_{A}, \Phi_{A}\right)+\left(\Phi_{A}, \Psi_{A}\right)$. Now $\left(\Psi_{A}, \Phi_{A}\right)=\omega\left(A^{*} A_{b}\right)$ $=\omega\left(A_{\frac{1}{2} b} * A_{\frac{1}{2} b}\right) \geqq 0$ and also $\left(\Phi_{A}, \Psi_{A}\right) \geqq 0$. Hence $\left\|\Psi_{A}\right\|^{2} \leqq\left\|\Psi_{A}+\Phi_{A}\right\|^{2}$ for all $A \in \widetilde{\mathcal{U}}$. It follows that the sequence $\Psi_{A_{n}}$ and the sequence $\Phi_{A_{n}}$ converge separately. Let $\chi_{1}=\lim _{n \rightarrow \infty} \Psi_{A_{n}}$ and $\chi_{2}=\lim _{n \rightarrow \infty} \Phi_{A_{n}}$. Clearly $\chi_{1}+\chi_{2}=\chi+J \chi^{*}$.

$$
\left(\chi-\chi_{1}, \Phi_{A}\right)=\left(\chi, \Phi_{A}\right)-\left(\chi_{1}, \Phi_{A}\right)=\left(J \chi^{*}, \Psi_{A}\right)-\lim _{n \rightarrow \infty}\left(\Psi_{A_{n}}, \Phi_{A}\right),
$$

where we used i. The invariance of $\omega(A)$ for the transformation $A \rightarrow A_{b}$ gives $\left(\Psi_{A}, \Phi_{B}\right)=\left(\Phi_{A}, \Psi_{B}\right)$ for all $A$ and $B \in \widetilde{\mathcal{A}}$. We have, therefore, $\left(\chi-\chi_{1}, \Phi_{A}\right)=\left(J \chi^{*}, \Psi_{A}\right)-\lim _{n \rightarrow \infty}\left(\Phi_{A_{n}}, \Psi_{A}\right)=\left(J \chi^{*}-\chi_{2}, \Psi_{A}\right)$ for all $A \in \widetilde{\mathfrak{A}}$. Since $\chi-\chi_{1}=\chi_{2}-J \chi^{*}$, we get $\left(J \chi^{*}-\chi_{2}, \Phi_{A}+\Psi_{A}\right)=0$ for all $A \in \widetilde{\mathfrak{A}}$, and hence $\chi_{2}=J \chi^{*}$ and $\chi_{1}=\chi$ or $\chi=\lim _{n \rightarrow \infty} \Psi_{A_{n}}$ and $J \chi^{*}=\lim _{n \rightarrow \infty} \Phi_{A_{n}}$; applying $J$ to both sides of the last equation we get $\chi^{*}=\lim _{n \rightarrow \infty} \Psi_{A_{n}^{*}}$.

iii. $\left(\Phi_{A}, R(\chi) S\left(\chi^{\prime}\right) \Phi_{B}\right)=\left(R(\chi)^{*} S\left(A^{*}\right) \Omega, S\left(\chi^{\prime}\right) \Phi_{B}\right)$

$$
=\left(\chi^{*}, S(A) S\left(\chi^{\prime}\right) \Phi_{B}\right)=\lim _{n \rightarrow \infty}\left(\Psi_{A_{n}}, S(A) S\left(\chi^{\prime}\right) \Phi_{B}\right)
$$

$=\lim _{n \rightarrow \infty}\left(R\left(A_{n}\right)^{*} \Omega, S(A) S\left(\chi^{\prime}\right) \Phi_{B}\right)=\lim _{n \rightarrow \infty}\left(S(A)^{*} \Omega, S\left(\chi^{\prime}\right) S\left(B^{*}\right) R\left(A_{n}\right) \Omega\right)$

$=\left(\Phi_{A}, S\left(\chi^{\prime}\right) S\left(B^{*}\right) \chi\right)=\left(\Phi_{A}, S\left(\chi^{\prime}\right) R(\chi) \Phi_{B}\right)$.

Consequently $\left(\Phi_{A},\left[R(\chi), S\left(\chi^{\prime}\right)\right] \Phi_{B}\right)=0$ for all $A, B \in \mathfrak{A}$, and thus $\left[R(\chi), S\left(\chi^{\prime}\right)\right]=0$.

${ }_{13}$ The proof given in a first draft of this work was not quite correct. In the proof presented here we made use of unpublished notes by D. KASTLER. 
The proof of theorem 4 follows now immediately. Combining the lemmas 4 and 5 we find that $\{S(\mathfrak{A})\}^{\prime} \subset\{R(\mathfrak{Q})\}^{\prime \prime}$. On the other hand, from lemma $3,\{R(\mathfrak{A})\}^{\prime \prime} \subset\{S(\mathfrak{Q})\}^{\prime}$; hence $\{R(\mathfrak{R})\}^{\prime \prime}=\{S(\mathfrak{A})\}^{\prime}$.

As in section 3 we construct the unitary operator which implements the automorphism of time-translation. We define the operator $\hat{U}(t)^{14}$ by the equation $\hat{U}(t) \Psi_{A}=\Psi_{A_{t}}$ for all $A \in \mathfrak{A}$. $\hat{U}(t)$ has the following properties:

1. $\hat{U}(t)$ is unitary.

2. $\hat{U}(t)$ commutes with $J$.

Proof. Let $A \in \widetilde{\mathfrak{A}} ; \hat{U}(t) \Phi_{A}=\hat{U}(t) \Psi_{A_{b}}=\Psi_{A_{b, t}}=\Psi_{A_{t, b}}=\Phi_{A_{t}}$. Since $\hat{U}(t)$ is bounded we have for all $A \in \mathfrak{A}: \hat{U}(t) \Phi_{A}=\Phi_{A_{t}}$. Now $\hat{U}(t) J \Psi_{A}$ $=\hat{U}(t) \Phi_{A *}=\Phi_{A *_{t}}=J \Psi_{A_{t}}=J \hat{U}(t) \Psi_{A}$; hence $[\hat{U}(t), J]=0$.

3. $\hat{U}(t) R(A) \hat{U}(t)^{-1}=R\left(A_{t}\right)$ and $\hat{U}(t) S(A) \hat{U}(t)^{-1}=S\left(A_{t}\right)$.

Proof. $\hat{U}(t) R(A) \hat{U}(t)^{-1} \Psi_{B}=\hat{U}(t) R(A) \Psi_{B_{-t}}=\hat{U}(t) \Psi_{A B_{-} t}=\Psi_{A_{t} B}$ $=R\left(A_{t}\right) \Psi_{B}$.

4. $\hat{U}(t) \Omega=\Omega$.

Like in the finite case, $\hat{U}(t)$ transforms both representations in the correct way. It is clear that $\hat{U}(t)$ belongs neither to $\{R(\mathfrak{Q})\}^{\prime \prime}$ nor to $\{S(\mathfrak{R})\}^{\prime \prime}$. The same holds for the infinitesimal generator $\hat{H}^{\prime}$, the hamiltonian.

In a similar way one can define a unitary operator $U(\alpha)$ for gauge transformations, and an unbounded self-adjoint generator $N$.

We also consider the operator $T$, defined by $T \Psi_{A}=\Phi_{A}$, with the following properties:

1. $T$ and $T^{-1}$ are unbounded self-adjoint positive definite operators.

2. For $A \in \widetilde{\mathfrak{A}} \quad T R(A) T^{-1}=R\left(A_{b}\right)$ and $T S(A) T^{-1}=S\left(A_{-b}\right)$.

Proof. The second statement is proved as follows. Let $A$ and $B \in \widetilde{\mathfrak{A}}$;

$$
\begin{aligned}
T R(A) T^{-1} \Psi_{B} & =T R(A) T^{-1} \Phi_{B_{-b}}=T R(A) \Psi_{B_{-b}}=T \Psi_{A B_{-b}}=\Phi_{A B_{-b}} \\
& =\Psi_{A_{b} B}=R\left(A_{b}\right) \Psi_{B} ;
\end{aligned}
$$

hence $T R(A) T^{-1}=R\left(A_{b}\right)$.

To prove the first statement we shall show first that $T$ is symmetric:

$$
\begin{aligned}
& \left(\Psi_{A}, T \Psi_{B}\right)=\left(\Psi_{A}, J \Psi_{B^{*}}\right)=\left(\Psi_{B^{*}}, J \Psi_{A}\right)=\left(\Psi_{B^{*}}, \Phi_{A^{*}}\right) \\
& \quad=\left(R\left(B^{*}\right) \Omega, S(A) \Omega\right)=\left(S\left(A^{*}\right) \Omega, R(B) \Omega\right)=\left(\Phi_{A}, \Psi_{B}\right)=\left(T \Psi_{A}, \Psi_{B}\right) .
\end{aligned}
$$

${ }^{14}$ As in section 3 the notation $U_{t}$ is reserved for the unitary operator for timetranslation in Fock space. 
In the second step we made use of property 3 of $J$. Since $T$ is symmetric the extension $T^{* *}$ is symmetric and closed. We can therefore assume that $T$ is closed. To prove that $T$ is self-adjoint we can make use of the Cayley transformation, $K=(T+i)(T-i)^{-1}$, and prove that $K$ is unitary, and hence $T=T^{*}$. We shall omit the proof, since property 1 follows immediately from the following equation:

$$
T=\exp \left\{-\frac{1}{2} \beta \hat{H}^{\prime}\right\}
$$

To prove (4.13) we define the self-adjoint operator $R=\exp \left\{-\frac{1}{2} \beta \hat{H}^{\prime}\right\}$ and we shall prove that $T=R$. The unbounded self-adjoint operator $\hat{H}^{\prime}$ has the spectral decomposition $\hat{H}^{\prime}=\int \varepsilon d E(\varepsilon)$ and hence

$$
R=\int e^{-\frac{1}{2} \beta \varepsilon} d E(\varepsilon) .
$$

Since $\exp \left\{i \hat{H}^{\prime} t\right\} \Psi_{A}=\Psi_{A_{t}}$, and thus

$$
\int \exp (i \varepsilon t) d E(\varepsilon) \Psi_{A}=\int \exp (-i \varepsilon t) \Psi_{\hat{A}(\varepsilon)} d \varepsilon,
$$

the measure $d E(\varepsilon) \Psi_{A}=\Psi_{\hat{A}(-\varepsilon)} d \varepsilon$ has compact support, for all $A \in \widetilde{\mathfrak{A}}$. Therefore, the operator (4.14) can be applied to $\Psi_{A}$ for $A \in \widetilde{\mathfrak{I}}$ and we find $R \Psi_{A}=\int \exp \left(-\frac{1}{2} \beta \varepsilon\right) d E(\varepsilon) \Psi_{A}=\Psi_{A}=\Phi_{A}$.

Let $D$ be the dense set of all $\Psi_{A}, A \in \widetilde{\mathfrak{A}}$, and let $T_{D}$ and $R_{D}$ be the restrictions of $T$ and $R$ on the domain $D$. Then we found

$$
T_{D}=R_{D} .
$$

Since $T$ and $R$ are symmetrical operators, the same holds for $T_{D}=R_{D}$. Clearly $T_{D}^{* *}=R_{D}^{* *}$ is a closed symmetric extension.

As we have seen for $A \in \widetilde{\mathfrak{A}}$ the measure $d E(\varepsilon) \Psi_{A}$ vanishes for $\varepsilon>\varepsilon_{0}$. Consider now

$$
\left\|R^{n} \Psi_{A}\right\|^{2}=\int \exp (-n \beta \varepsilon)\left(\Psi_{A}, d E(\varepsilon) \Psi_{A}\right) \leqq \exp \left(-n \beta \varepsilon_{0}\right)\left\|\Psi_{A}\right\|^{2} .
$$

Hence the power series $\sum_{n} z^{n}(n !)^{-1}\left\|R^{n} \Psi_{A}\right\|$ has a finite radius of convergence, so that $\Psi_{A}, A \in \widetilde{\mathfrak{A}}$, is an analytic vector of $R$, as defined by NeLson [14]. The operator $R_{D}^{* *}=T_{D}^{* *}$ is a closed symmetric operator with a dense set of analytic vectors. As a consequence of a theorem due to NELSON ${ }^{15}$, this operator is self-adjoint. Now $T$ and $R$ are both closed symmetric extensions of $T_{D}^{* *}=R_{D}^{* *}$ and hence $T=R$.

15 Reference [14] lemma 5.1. 
We shall end this section by reconsidering condition II on $\omega(A)$. Let us therefore see what one would obtain without making use of this property. It turns out that almost all results, obtained in this section, in particular theorem 4, remain valid. There are however the following modifications:

1. Let $J$ be the left ideal of all $A \in \mathfrak{A}$ for which $\omega\left(A^{*} A\right)=0$. Then $\Psi_{C}$ is the null vector for $C \in J$, and $\Psi_{A}=\Psi_{B}$ if $A-B \in J$.

2. $R(C)=0$ for $C \in J$. This follows from lemma 2 and definition (4.11). Consequently the representation is not faithful, and the kernel $J$ is a two-sided *-ideal of $\mathfrak{A}$. For a simple algebra $J$ consists of the zero element alone.

\section{Conclusion}

We have seen that the symmetry between the representative of the algebra and its commutant, as found first by ARAKI and WooDs for the free Bose gas, is a general feature of states in thermal equilibrium at a given $T$ and $\mu$. At the present time the physical significance is still unclear.

It is interesting to note that the symmetry is not restricted to equilibrium states. To make this clear we point out that in section 3 we assumed the existence of an automorphism $A \rightarrow A_{t}$ for which $\omega(A)$ is invariant. It then follows that there exists a unitary operator $\hat{U}(t)$ $=\exp (i \hat{H} t)$ on $\mathfrak{G}$, which implements this automorphism. This does not mean, however, that the system actually moves according to this automorphism. It only means that it is possible to choose the dynamics, i.e. the interparticle forces and the external forces, such that with these forces the system in the state $\omega(A)$ would be in equilibrium. If the forces happen to be different, the automorphism $A \rightarrow A_{t}$ is not a time translation, $H$ is not the Hamiltonian of the system and the state $\omega(A)$ is not stationary, but the symmetry between the representative of the algebra and its commutant still exists.

Acknowledgements. The authors would like to thank Professors D. Ruelle, D. Kastler, D. W. Robinson and H. J. Borchers for illuminating discussions. One of the authors (N. M. H.) would like to express his indebtedness to Professor ALMY for his hospitality at the University of Illinois, where this work initiated, to Professor L. Motchane for the hospitality extended to him at the I.H.E.S. at Bures-sur-Yvette and to Professor Pond for his invitation to join the Summer Symposium on theoretical physics at the State University of New York at Stony Brook, where a first draft of this work was written.

\section{References}

1. Kubo, R.: J. Phys. Soc. Japan 12, 570 (1957).

2. Martin, P. C., and J. Schwinger: Phys. Rev. 115, 1342 (1959).

3. Araki, H., and E. J. Woods: J. Math. Phys. 4, 637 (1963). 
4. Doplicher, S., D. Kastler, and D. W. Robinson: Commun. Math. Phys. $\mathbf{3}, 1$ (1966).

5. Ruelle, D.: Commun. Math. Phys. 3, 133 (1966).

6. Kastler, D., and D. W. Robinson: Commun. Math. Phys. 3, 151 (1966).

7. HAAG, R.: Lecture Notes, Pacific Summer School, Hawaii (1965).

8. Araki, H., and W. Wyss: Helv. Phys. Acta 37, 136 (1964).

9. Dell Antonio, G. F., S. Doplicher, and Ruelle D.: Commun. Math. Phys. 2, 223 (1966).

10. Ruelle, D.: Helv. Phys. Acta 36, 789 (1963).

11. Dyson, F. J., and A. Lenard: Preprint.

12. Dixmier, J.: Les algèbres d'operateurs dans l'espace Hilbertien (Algèbres de von Neumann). Paris: Gauthier-Villars 1957.

13. Segal, I.E.: Mat. Fys. Medd. Dan. Vid. Selsk. 31, 12 (1959).

14. Nelson, E.: Ann. Math. 70, 572 (1959). 